\title{
LOOKING FOR THE FIFTH DIMENSION
}

\author{
Paul S. Wesson \\ Department of Physics and Astronomy, University of Waterloo, Waterloo, Ontario N2L 3G1, Canada
}

Received 2014-02-18; Revised 2014-02-18; Accepted 2014-03-19

\begin{abstract}
An exact solution shows that a new kind of wave can propagate through the Einstein vacuum if there is an extra dimension, thereby providing a way to test the dimensionality of the universe.

Keywords: Extra Dimensions, Cosmological Constant, Causality
\end{abstract}

\section{INTRODUCTION}

Einstein and Bergmann (1938), working with Bergmann, stated: "We ascribe physical reality to the fifth dimension".

Since that time, the fortunes of general relativity a la Kaluza and Klein have waxed and waned. Today, many researchers feel that extra dimensions offer the most promising route to an eventual unification of gravity with the interactions of particles. But modern versions of the theory suffer from their own modesty: They reduce in the appropriate limit to four-dimensional general relativity, which ensures their agreement with observation but also leads to merely minor and unexciting differences. What is needed is some new kind of consequence of 5D, which will highlight fresh physics not to be found in 4D.

In this study, we present a solution to the $5 \mathrm{D}$ field equations with unusual properties (Wesson, 2013). It describes a new kind of wave-not gravitational or electromagnetic-which can propagate superluminally and depends for its existence on the vacuum. This solution, which may be checked by computer, most resembles the de Broglie matter waves of old quantum theory. In the absence of a proper theory of quantum gravity, this solution may represent a tentative step towards the unification of physics at the large and small scales.

The field equations of five-dimensional relativity are commonly taken to be given by the Ricci tensor as $\mathrm{R}_{\mathrm{AB}}=$ $0(\mathrm{~A}, \mathrm{~B}=0,123,4)$ for time, ordinary space and the extra coordinate). These 5D equations actually contain Einstein's 4D equations, by Campbell's embedding theorem (Seahra and Wesson, 2003). It is a corollary of this theorem that when the 5D metric takes the so-called canonical form, it embeds all solutions of general relativity which are empty of ordinary matter but contain finite vacuum energy as measured by the cosmological constant (Romero et al., 1996; Mashhoon et al., 1994). The latter parameter $\Lambda$ is positive or negative, depending on whether the extra dimension is space like or time like. For both cases, the equation of state of the vacuum in terms of its effective energy density and pressure is $p_{v}=$ $-\rho_{\mathrm{v}}=-\Lambda / 8 \pi$. Here units have been chosen which render the speed of light $c$ and the gravitational constant $G$ unity, though these quantities and Planck's constant $h$ will be made explicit later in order to aid physical understanding. The extra coordinate will be labelled $\mathrm{x}^{4}=$ 1 , to avoid confusion with the usual coordinates of space time $x^{a}=t, x y z$. Other notation is standard.

There are many solutions known of the $5 \mathrm{D}$ field equations $\mathrm{R}_{\mathrm{AB}}=0$, including several in canonical form (Wesson, 2013; Seahra and Wesson, 2003; Romero et al., 1996; Mashhoon et al., 1994). The 5D Schwarzschild-de Sitter solution is one such, which ensures the agreement of 5D relativity with the classical tests of Einstein's theory. But for present purposes, consider the following solution Equation 1:

$$
d S^{2}=\frac{1^{2}}{L^{2}}\left\{\begin{array}{l}
\mathrm{dt}^{2}-\exp \left[ \pm \frac{2 i}{\mathrm{~L}}(\mathrm{t}+\alpha \mathrm{x})\right] \mathrm{dx}^{2} \\
-\exp \left[ \pm \frac{2 \mathrm{i}}{\mathrm{L}}(\mathrm{t}+\beta \mathrm{y})\right] \mathrm{dy}^{2} \\
-\exp \left[ \pm \frac{2 \mathrm{i}}{\mathrm{L}}(\mathrm{t}+\gamma \mathrm{z})\right] \mathrm{dz}^{2}
\end{array}\right\}+\mathrm{dl}^{2}
$$


This describes a wave propagating through ordinary $3 \mathrm{D}$ space, where the frequency $\mathrm{f}=1 / \mathrm{L}$ is fixed by the solution. The wave-numbers $\mathrm{k}_{\mathrm{x}}=\alpha / \mathrm{L}, \mathrm{k}_{\mathrm{y}}=\beta / \mathrm{L}, \mathrm{k}_{\mathrm{z}}=\gamma / \mathrm{L}$ along the $\mathrm{x}, \mathrm{y}, \mathrm{z}$ axes are written in terms of the dimensionless constants $\alpha, \beta, \gamma$ which are arbitrary. The phase velocity of the wave along (say) the $\mathrm{x}$-axis is $\mathrm{c} / \alpha$ and is also arbitrary. The constant length $L$ in (1) is likewise arbitrary in a mathematical sense, but plays on important physical role. It was originally introduced to the canonical metric as a measure of the scale of the 4D potential. But in vacuum spacetimes, this is given by the $4 \mathrm{D}$ curvature which is provided by the cosmological constant $\Lambda$. By evaluating the Einstein tensor for the 4D part of (1), it may be shown by some algebra that the equations of general relativity are satisfied, with a cosmological constant $\Lambda$ $=-3 / \mathrm{L}^{2}$. Accordingly, (1) describes waves travelling in a classical vacuum with positive pressure.

Other properties of (1) may be revealed by using one of the software packages currently available. In fact, while (1) was found originally by solving the field equations by hand, the quickest way to verify it is by computer.

Perhaps the most striking feature of (1) is that it allows phase velocities that are, in a formal sense, greater than the speed of light. This because, as noted above, the phase speed is c/ $\alpha$ and $\alpha$ can be arbitrarily small. It should be recalled that this does not necessarily conflict with causality, as long as the waves concerned do not carry conventional information (Bell, 2004); and there is a (somewhat unusual) interpretation of the Lorentz transformations due to Rindler that allows such speeds (Rindler, 1977). The fact that the waves described by (1) can be superluminal implies that they are not conventional gravitational waves of the kind found in 4D general relativity. This is confirmed by noting that the waves in (1) depend for their existence on the presence of vacuum energy with its typical equation of state $\left(\mathrm{p}_{\mathrm{v}}=\right.$ $-\rho_{\mathrm{v}}$ ), whereas standard gravitational waves propagate through truly empty space $(p=\rho=0)$. Also, in the quantum version of classical Kaluza-Klein theory, the particles associated with the scalar field have spin 0 , while in Einstein's theory the graviton has spin 2. In understanding the possibility of superluminal velocities as given by the solution (1) of 5D relativity, it is especially useful to consider 4D wave mechanics.

The theoretical basis of de Broglie waves was stated by him in 1924 . He postulated that any massive particle has associated with it a wave, in analogy with a photon's electromagnetic wave, in a way that respects the Lorentz transformations and Planck's law. The direction of motion of the particle is the same as the normal to the wave-front. Let the mass and velocity of the particle be $\mathrm{m}, \mathrm{v}$ and the frequency and velocity of the wave be $\mathrm{f}$, w. Then in terms of the 4-vectors for the particle and the wave, there is a match of the magnitude of the quantities concerned, via de Broglie's equation: $m$ $(\mathrm{v}, 1)=\mathrm{hf}\left(1 / \mathrm{w}, 1 / \mathrm{c}^{2}\right)$. Equating components in de Broglie's equation gives $\mathrm{mv}=\mathrm{hf} / \mathrm{w}$ and $\mathrm{m}=\mathrm{hf} / \mathrm{c}^{2}$. The second of these relations is simply a statement of the equality of the energy of the particle and the energy of the wave: $E=\mathrm{mc}^{2}=\mathrm{hf}$. The first relation, when divided by the second, gives $\mathrm{vw}=\mathrm{c}^{2}$.

To see how this relation compares with the wave of the solution (1), consider the element of proper distance in the latter along the $\mathrm{x}$-axis, given by $\mathrm{d} \overline{\mathrm{x}}=\exp \left[\mathrm{i}\left(\mathrm{ft}+\mathrm{k}_{\mathrm{x}} \mathrm{x}\right)\right] \mathrm{dx}$. The frequency is $\mathrm{f}=\mathrm{c} / \mathrm{L}$. By Planck's law, the energy of the wave is equivalent to the mass $m$ of the associated particle, $\mathrm{E}=\mathrm{hf}=\mathrm{hc} / \mathrm{L}=\mathrm{mc}^{2}$, so $\mathrm{L}=\mathrm{h} / \mathrm{mc}$. That is, the size of the $4 \mathrm{D}$ potential well in (1) equals the Compton wavelength of the particle. The momentum of the particle $\mathrm{p}_{\mathrm{x}}=\mathrm{mv}_{\mathrm{x}}$ is inversely proportional to the wavelength, so the wave-number $\mathrm{k}_{\mathrm{x}}$ is directly proportional to $\mathrm{p}_{\mathrm{x}}$ and can be written in the correct dimensional form as $\mathrm{k}_{\mathrm{x}}=(\mathrm{mc} / \mathrm{h})\left(\mathrm{v}_{\mathrm{x}} / \mathrm{c}\right)=\mathrm{v}_{\mathrm{x}} / \mathrm{cL}$. Recalling that the frequency is $\mathrm{f}=\mathrm{c} / \mathrm{L}$, the phase velocity of the wave along the $\mathrm{x}$-axis is $\mathrm{w}_{\mathrm{x}}=\mathrm{f} / \mathrm{k}_{\mathrm{x}}=$ (c/L) $\left(\mathrm{cL} / \mathrm{v}_{\mathrm{x}}\right)=\mathrm{c}^{2} / \mathrm{v}_{\mathrm{x}}$. Thus along each axis, the velocities of the particle and the wave are connected by de Broglie's relation $\mathrm{vw}=\mathrm{c}^{2}$.

There are other areas of 'overlap' between wave motion in 5D and wave mechanics in 4D (Wesson, 2013; 2011). For example, the components of the geodesic equation in $5 \mathrm{D}$ yield in combination a scalar relation which is the same as the Klein-Gordon equation for the wave function in 4D. Also, the null path for the $5 \mathrm{D}$ interval defined by $\mathrm{dS}^{2}=0$ contains the usual paths for massive particles and photons in $4 \mathrm{D}$ as specified by $\mathrm{ds}^{2} \geq 0$. In other words, standard dynamics is preserved and the definition of causality is broadened by the extension to 5D. The implication is that the puzzling properties of de Broglie waves in $4 \mathrm{D}$ are due to truncating a well-behaved solution in 5D.

De Broglie waves are sometimes viewed with suspicion, largely because they entail velocities that exceed light speed. However, waves of this type arise inevitably in any 5D theory of the kind used in unification, if the extra coordinate is time like. Consider flat 5D space with line element $\mathrm{dS}^{2}=\mathrm{c}^{2} \mathrm{dt}^{2}$ $\left(d x^{2}+d y^{2}+d z^{2}\right)+d l^{2}$. For the 5D null-path $d^{2}=0$, the 
apparent velocity along the $\mathrm{x}$-axis has magnitude $\mathrm{dx} / \mathrm{dt}$ $=\left[\mathrm{c}^{2}(\mathrm{dl} / \mathrm{dt})^{2}\right]^{1 / 2}$ and can exceed $c$. For more realistic situations, in which there is a significant vacuum measured by a cosmological constant $\Lambda<0$, superluminal waves are a natural consequence. From the viewpoint of 5D field theory, waves of de Broglie type have to be considered real.

\section{CONCLUSION}

Not be surprising to those involved in modern experiments on the wave nature of matter (Kocsis et al., 2011). Recent refinements of the classic double-slit experiment and other setups, have confirmed the wave nature of matter for relatively large molecules (e.g., carbon-60). For a composite object, the de Broglie wavelength depends on its constituents and there is a regular transition of the wave properties from massive particles to entangled photons. Yet despite extensive experimental work, the origin of these waves is not well understood from the theoretical side. What has been shown here is that waves of de Broglie type can be regarded as the natural manifestation of a higher dimension of the kind needed for the unification of the interactions of physics. If such vacuum waves were present in the early universe, or exist in certain environments today, they could provide a way to test for the fifth dimension.

\section{ACKNOWLEDGEMENT}

Thanks go to J.M. Overduin for checking the solution (1) of the text on the computer.

\section{REFERENCES}

Einstein, A. and P. Bergmann, 1938. On a generalization of Kaluza's theory of electricity. Ann. Math., 39: 683-701. DOI: 10.2307/1968642

Bell, J.S., 2004. Speakable and Unspeakable in Quantum Mechanics. 1st Edn., Cambridge University Press, Cambridge, ISBN-10: 0521523389, pp: 248.

Kocsis, S., B. Braverman, S. Ravets, M.J. Stevens and R.P. Mirin et al., 2011. Observing the average trajectories of single photons in a two-slit interferometer. Science, 332: 1170-1173. DOI: 10.1126/science. 1202218

Mashhoon, B., H. Liu and P.S. Wesson, 1994. Particle masses and the cosmological constant in KaluzaKlein theory. Phys. Lett. B, 331: 305-312. DOI: 10.1016/0370-2693(94)91057-X

Rindler, W., 1977. Essential Relativity: Special, General and Cosmological. 2nd Edn., Springer, New York, ISBN-10: 038707970X, pp: 284.

Romero, C., R. Tavakol and R. Zalaletdinov, 1996. The embedding of General Relativity in five dimensions. Gen. Rel. Gravit., 28: 365-376. DOI: 10.1007/BF02106973

Seahra, S.S. and P.S. Wesson, 2003. Application of the Campbell-Magaard theorem to higher-dimensional physics. Class. Quant. Gravity, 20: 1321-1321. DOI: 10.1088/0264-9381/20/7/306

Wesson, P.S., 2011. General relativity and quantum mechanics in five dimensions. Phys. Lett. B, 701: 379-383. DOI: 10.1016/j.physletb.2011.05.038

Wesson, P.S., 2013. Vacuum waves. University of Waterloo. 